\title{
ABSTRACT \\ A LONGITUDINAL EXAMINATION OF THE RELATIONSHIP BETWEEN EATING DISORDER SYMPTOMS AND SUICIDAL IDEATION
}

\author{
by Shelby N. Ortiz
}

There is an assumption that eating disorders (EDs) serve as a risk factor for - and therefore, precede - suicidal thoughts and behaviors (STBs). However, because most studies investigating the ED-STB link have been limited to cross-sectional or retrospective designs, this assumption has not been adequately tested. There are other limitations with previous work that must be addressed. First, the mean follow-up time for the few longitudinal studies that exist was nearly a decade. Moreover, only one study has examined whether an ED factor (i.e., diagnosis or symptom) longitudinally predicts suicidal ideation. Even within cross-sectional studies, suicidal ideation has been poorly measured, with most studies using only one item to assess suicidal ideation. Thus, the present study used autoregressive cross-lagged modeling to test if a bidirectional relationship exists between ED symptoms and suicidal ideation among an ED patient sample over five weekly time points. Results revealed that suicidal ideation and ED symptoms were associated with each other at each time point. Additionally, suicidal ideation at Week 3 predicted ED symptoms at Week 4, while controlling for Week 3 ED symptoms. Given that suicidal ideation may influence future engagement in ED symptoms, targeting suicidal thoughts in therapy may help to reduce eating pathology. 


\title{
A LONGITUDINAL EXAMINATION OF THE RELATIONSHIP BETWEEN EATING DISORDER SYMPTOMS AND SUICIDAL IDEATION
}

\author{
Thesis \\ Submitted to the \\ Faculty of Miami University \\ in partial fulfillment of \\ the requirements for the degree of \\ Master of Arts \\ Department of Psychology \\ by \\ Shelby N. Ortiz \\ Miami University \\ Oxford, Ohio \\ 2019 \\ Advisor: April R. Smith, Ph.D. \\ Reader: Aaron M. Luebbe, Ph.D. \\ Reader: Elise M. Clerkin, Ph.D.
}


This thesis report titled

\title{
A LONGITUDINAL EXAMINATION OF THE RELATIONSHIP BETWEEN EATING DISORDER SYMPTOMS AND SUICIDAL IDEATION
}

by

\author{
Shelby N. Ortiz \\ has been approved for publication by \\ The College of Arts and Sciences \\ and \\ Department of Psychology
}

April R. Smith, Ph.D.

Aaron M. Luebbe, Ph.D.

Elise M. Clerkin, Ph.D. 


\section{Table of Contents}

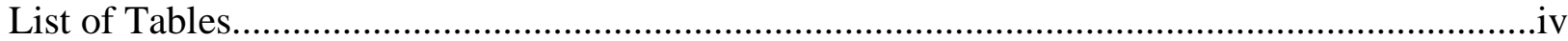

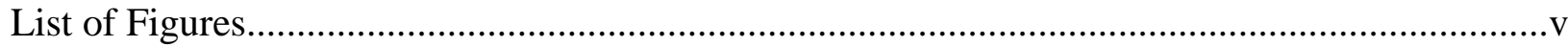

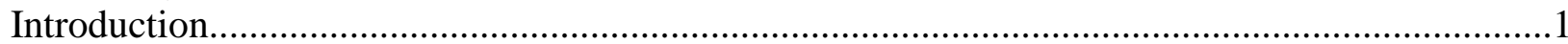

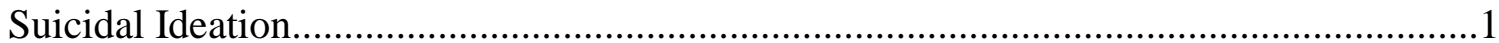

The Directionality of the ED-STBs Association............................................................

EDs Contribute to suicidal ideation risk..............................................................

Suicidal ideation contributes to ED symptoms......................................................

EDs and STBs contribute to each other bi-directionally.........................................

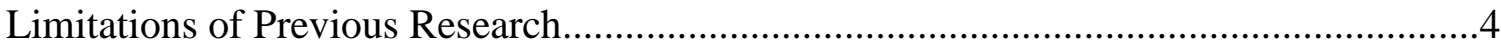

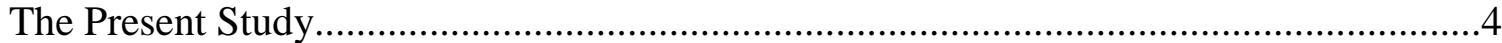

Method....

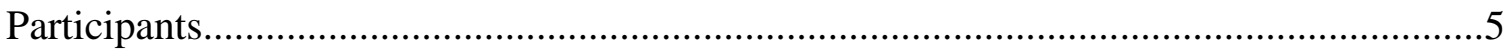

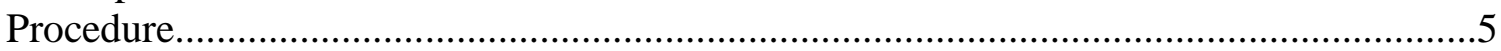

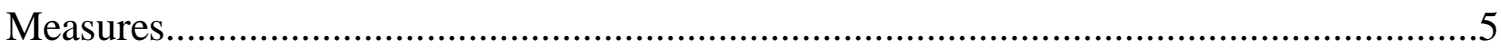

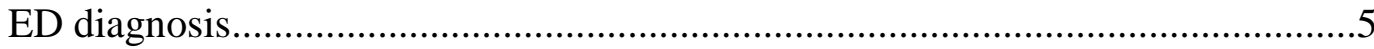

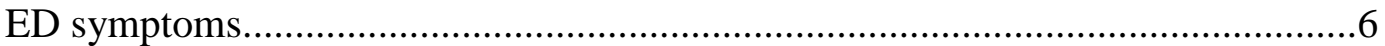

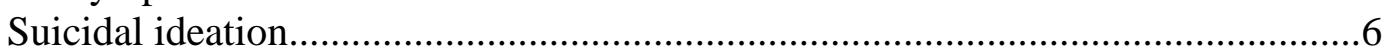

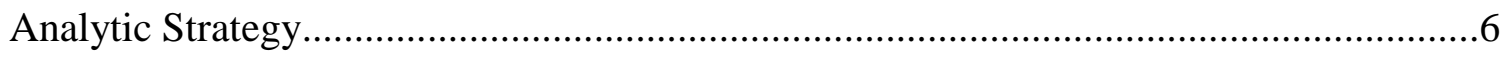

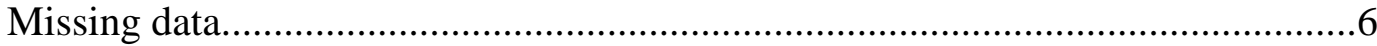

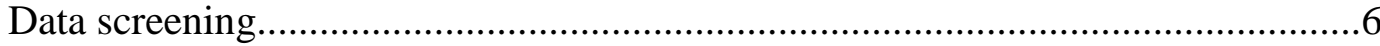

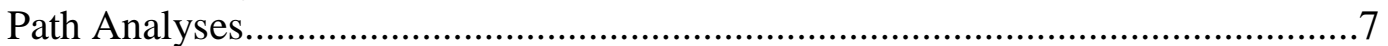

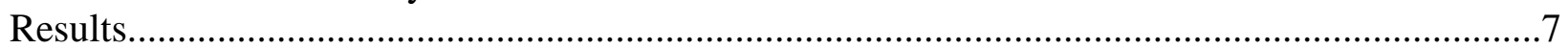

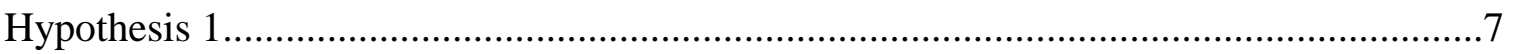

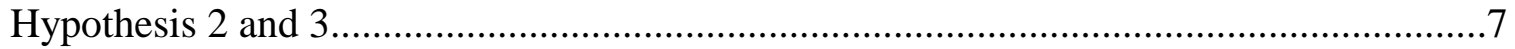

Eating concerns......................................................................................

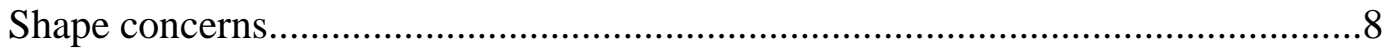

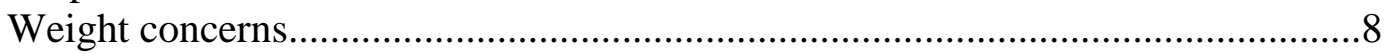

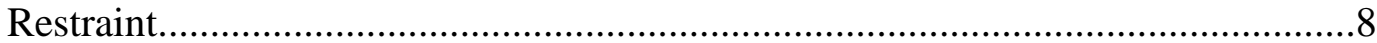

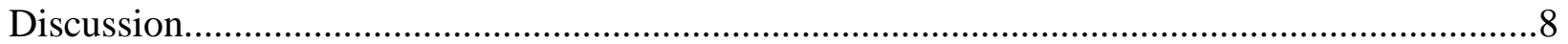

Strengths, Limitations, and Future Directions...............................................................10

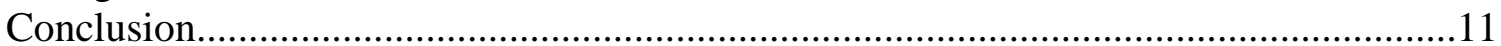

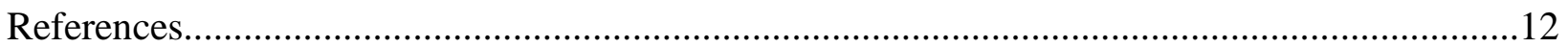




\section{List of Tables}

Table 1. Descriptive Statistics and Intercorrelations between Study Measures..........................18

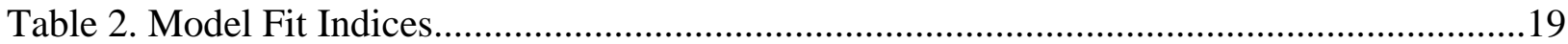




\section{List of Figures}

Figure 1. Autoregressive Cross-Lag Model Tested ..................................................................20 


\section{Introduction}

Eating disorders (EDs) are robustly associated with suicidal thoughts and behaviors (STBs; Brausch \& Gutierrez, 2009; Favaro \& Santonastaso, 1997; Herpertz-Dahlmann et al., 2008). Clinical and epidemiological studies confirm that ED patients report elevated rates of suicidal ideation, attempts, and death compared to the general public (Arcelus et al., 2011; Chesney, Goodwin, \& Fazel, 2014; Nock et al., 2008). However, our understanding of the dynamic interplay between these conditions remains limited (Smith, Zuromski, \& Dodd, 2018). The need to understand the relationship between EDs and STBs is crucial given that our ability to predict who will die by suicide is barely above chance accuracy (Franklin et al., 2017). Although suicidal ideation is a precursor to suicidal behavior (Rodzinski, Rutkowski, \& Ostachowska, 2017), it appears to be one of the most understudied types of STBs within ED populations (Smith, Zuromski, \& Dodd, 2018). In fact, longitudinal research examining suicidal ideation in those with EDs is almost non-existent. In order to increase our understanding of the relationship that exist between these conditions, the proposed study explored how ED symptoms and suicidal ideation related to each other across multiple time points.

\section{Suicidal Ideation}

Suicidal ideation, or suicidal thoughts, can range from a fleeting consideration of death to a detailed plan for suicide. The onset of suicidal ideation usually occurs during adolescence (Hawton \& van Heeringen, 2009). Known risk factors for suicidal ideation include having a psychiatric disorder (especially depression and substance abuse), psychological characteristics such as loneliness, aggression, impulsivity, and negative life events, and genetic and biological factors (Hawton \& van Heeringen, 2009). Experiencing suicidal ideation during adolescence has been linked to negative subsequent mental health outcomes in adulthood such as increased reports of depression, substance use, suicide attempts, and an overall decrease in well-being (Fergusson, Horwood, Ridder, \& Beautrais, 2005). Thus, it is pertinent that we continue to identify additional risk factors for - and the consequences of - suicidal ideation to prevent escalation to more severe forms of suicidality.

\section{The Directionality of the ED-STBs Association}

Research investigating the degree to which suicidal ideation is associated with EDs suggests a robust relationship. Whereas the general population experiences suicidal ideation at a rate between 5.6-14.4\% (Nock et al., 2008), one-third to one-fourth of those with EDs experience lifetime suicidal thoughts (Cararno et al., 2012; Favaro \& Santonastaso, 1997; Milos et al., 2004). Although these rates illustrate that there is a connection between ED symptoms and STBs, much is still unknown about the possible pathways by which these conditions are related to each other (Smith, Zuromski, \& Dodd, 2018). The pathway that has been the primary focus of theories and research is that ED symptoms directly or indirectly contribute to STBs. However, more recent research suggests that STBs may directly or indirectly contribute to disordered eating (Forrest et al., 2016; Trujillo et al., under review). Further yet, there may be a bi-directional relationship, whereby EDs and STBs influence one another over time. Currently, research on possible bi-directional relations between EDs and suicidal ideation has yet to be conducted. Below, theories and research findings that support each of these possible pathways are discussed in turn.

EDs contribute to suicidal ideation risk. The Interpersonal Theory of Suicide (IPTS; Joiner, 2005; Van Orden et al., 2010) offers a persuasive framework to explain why disordered eating may lead to the onset of STBs. The theory argues that in order for one to die by suicide, an individual must desire death, as well as have the capability to make a lethal attempt. Those that 
feel like a burden to others around them and like they do not belong are at risk for desiring death (i.e., active suicidal ideation). When individuals have an elevated pain tolerance and a fearlessness about death, this contributes to one having the capability to attempt suicide. In sum, the IPTS proposes that suicidal desire and capability for suicide must converge for dangerous suicidal behavior to occur.

Various researchers have used the IPTS as a framework to test whether ED symptoms are related to burdensomeness, low belongingness, and/or capability for suicide. For instance, Smith, Witte, and Crow (2014) hypothesized that EDs may lead to feelings of burdensomeness due to the financial and interpersonal costs resulting from the disorders (Zabala et al., 2009; Sepulveda et al., 2014). Beyond the treatment costs, which can quickly exceed thousands of dollars (Frisch, Herzog, \& Franko, 2006), people with EDs are less likely to be employed than those without EDs (Samnaliev, Noh, Sonneville, \& Austin, 2015). This then can lead to shame and a perceived liability among ED patients (Troop, Allan, Serpell, \& Treasure, 2008), increasing the risk for those with EDs to feel like a burden to others.

With regard to low belongingness, individuals with EDs often have disturbed psychosocial relationships (Bohn et al., 2008) that can lead to fewer social connections (Fairburn et al., 1999), elevated loneliness (Harney et al., 2014), and reduced ability to experience pleasure from relationships with others (Harrison, Mountford, \& Tchanturia, 2014; Tiller et al., 1997). Engaging in ED behaviors can also be extremely isolating as these behaviors might lead to rigid workout schedules, furtive eating, and other behaviors that are done in secret, such as purging (Rieger et al., 2010; Dodd, Smith, \& Bodell, 2014; Keys et al., 1950; Forrest et al., 2016). This multitude of interpersonal difficulties can contribute to feelings of burdensomeness and thwarted belongingness, which together lead to the desire for suicide.

A handful of studies have directly examined thwarted belongingness and perceived burdensomeness within a sample of individuals engaging in ED behaviors or those with an ED diagnosis. Within an ED sample, Smith and colleagues (2016) found that burdensomeness (but not thwarted belongingness) was related to suicidal ideation, and both burdensomeness and thwarted belongingness were greater in the ED sample than a control group. Similarly, Pisetsky and colleagues (2017) found that ED symptoms were related to burdensomeness and low belongingness within another ED sample. Among a nonclinical sample, Dodd, Smith, and Bodell (2014) found that dietary restraint (but not bingeing, purging, body satisfaction, or drive for thinness) indirectly predicted low belongingness and perceived burdensomeness via the pathway of negative life events. Although these studies offer some support of the IPTS, a later section will detail how several other longitudinal studies have found an association in the opposite direction of the relationship (i.e., that STBs precede EDs).

The capability to attempt suicide is believed to develop through habituation to pain and fear after repeated exposure to painful and provocative events (Joiner, 2005; Van Orden et al., 2010). Researchers have hypothesized that ED behaviors (e.g., restriction of food, overexercising, vomiting) may serve as painful and provocative events that lead to increased capability for suicide (Selby et al., 2010; Smith, Yeager, \& Dodd, 2015). Although the development of the capability for suicide is beyond the scope of this study (given the focus on suicidal ideation), it is worth mentioning that while some cross-sectional studies find an association between ED behaviors and capability for suicide (Smith et al., 2016), others do not (e.g., Zuromski \& Witte, 2015).

While support for using the IPTS as a framework for understanding STBs in EDs is mixed, other studies do suggest that EDs may serve as risk factors for STBs (Smith, Velkoff, 
Riberiro, \& Franklin, 2018; Yao et al. 2016). Of note, a meta-analysis that reviewed the longitudinal research between EDs and STBs found that having an ED diagnosis or experiencing ED symptoms were significant but weak predictors of suicide attempts, but not suicide death (Smith et al., 2018). Taken together, there is some work that supports the notion that certain ED symptoms precede STBs. However, the limited support comes mostly from cross-sectional research. It is evident that more longitudinal research is needed to conclusively determine if this directionality best describes the relationship between EDs and suicidal ideation.

Suicidal ideation contributes to ED symptoms. If viewed from the standpoint that maladaptive eating behaviors are used to cope with experiences of negative emotion (Leehr et al., 2015), it is conceivable that suicidal ideation may lead some individuals to rely on ED behaviors as a way to escape from their thoughts about suicide (Paxton \& Diggens, 1997; Wildes et al., 2010). The interpersonal model of ED pathology, which will be discussed in more detail in a later section, outlines how specific ED behaviors are used to escape aversive states and offers additional support for the proposition that this type of relationship between ED symptoms and suicidal ideation may exist.

Although a limited number of studies have tested if suicidal ideation contributes to individuals engaging in ED symptoms, several longitudinal studies do support the idea that the relation could flow in this direction. Researchers have found that low social support from family and peers predicted increases in body dissatisfaction among males and females (Bearman, Presnell, Martinez, \& Stice, 2006; Stice \& Whitenton, 2002). Further, Stice, Presnell, and Spangler (2002) found that perceiving low social support from peers and having low self-esteem at Time 1 were risk factors for the onset of binge eating two years later. Overweight individuals are likely to experience loneliness due to reduced social support and negative evaluation from others and they might binge eat in response (Almeida, Savoy, \& Boxer, 2011; Ashmore, Friedman, Reichmann, \& Musante, 2008). Within a sample of individuals with EDs, Trujillo et al. (under review) found that over an eight-week period, ED symptoms did not predict change in thwarted belongingness or perceived burdensomeness, but perceived burdensomeness positively predicted increases in ED symptoms. Additionally, Forrest and colleagues (2016) found that suicidal ideation, suicide planning, and suicide attempts generally preceded binge eating disorder (BED) onset among a large, nationally representative sample of adults. While it seems plausible that risk factors for suicidal ideation, such as low belongingness and perceived burdensomeness, can lead to the use of ED symptoms as a way to cope, not enough research has directly examined whether suicidal ideation precedes the onset of EDs.

EDs and STBs contribute to each other bi-directionally. Lastly, it could be that ED symptoms and STBs are actually bi-directionally related. Although this directionality has not been directly tested, there are theories that lend support to this relationship. The interpersonal model of ED pathology (Rieger et al., 2010) maintains that ED behaviors and symptoms are exhibited when individuals experience negative interpersonal interactions, have negative selfevaluation (i.e., negative beliefs regarding one's worth), and have poor self-regulation (i.e., use ineffective strategies to regulate mood). Engagement in ED behaviors increasingly replaces healthy engagement with the social world, which in turn, exacerbates interpersonal problems. For instance, dieting may be used to increase self-esteem (Cooper et al., 2004), binge eating may be used to escape aversive states of self-awareness (Heatherton \& Baumeister, 1991), and selfstarvation may be used to avoid any feelings at all (Schmidt \& Treasure, 2006). As previously discussed, routinely engaging in ED symptoms can cause an individual to be at additional risk for suicidal ideation by increasing their social isolation and feelings of burdensomeness (Dodd, 
Smith \& Bodell, 2014; Forrest et al., 2016; Keys et al., 1950; Rieger et al., 2010). The individual is then vulnerable to experiencing additional suicidal thoughts and/or behaviors, perpetuating the cycle. Thus, the aim of the present study is to test if this model explains the ED-suicide link by examining if a bi-directional relationship exists between ED symptoms and suicidal ideation in an ED sample.

\section{Limitations of Previous Research}

In addition to the unresolved issue of directionality, there are other limitations with previous work that must be addressed. The majority of STBs research has been studied in those with AN, with fewer studies on BN, BED, and other specified feeding/eating disorder (OSFED). Minimal work has made use of the $5^{\text {th }}$ edition of the Diagnostic and statistical manual of mental disorders (DSM; American Psychiatric Association, 2013) diagnostic criteria in general, making it challenging to know if changes from the DSM-IV-TR to the DSM-5 criteria for ED diagnoses affect STBs rates.

A meta-analysis conducted by Smith and colleagues (2018) highlights other shortcomings of the research on this topic. At the time of the meta-analysis, no studies examining whether an ED factor longitudinally predicted suicidal ideation had been published. Additionally, Smith et al.'s (2018) meta-analysis found that the mean follow-up time for studies predicting attempts or suicide death was nearly a decade, contributing to a lack in understanding which ED behaviors might be short-term predictors for STBs and vice versa. Fluctuations in affect can influence the experience of suicidal ideation over the course of a week (e.g., Links et al., 2007; Nock et al., 2009). Similarly, other work has found that engagement in ED behaviors can also change on a weekly basis for individuals in treatment (e.g., Linardon, Brennan, \& De laPiedad Garcia, 2016). Thus, this initial step of using weekly follow-up points has the possibility of capturing whether changes in ideation or disordered eating symptoms influence one another.

The measurement of suicidal ideation among those with EDs is an additional area that requires improvement. Since the publication of Smith et al.'s (2018) meta-analysis, one recent study did observe changes in suicidal ideation rates within a sample of AN patients that were not experiencing suicidal ideation at time of admission into treatment (Bodell, Cheng, \& Wildes, 2018). These patients later experienced suicidal ideation at rates between $13.6-16.7 \%$ at the three-, six-, and 12-month follow-up time points. However, this study was limited by using only one item to assess current suicidal ideation. Even within cross-sectional studies, suicidal ideation has been poorly measured, with the majority of studies using a single-item assessment of suicidal ideation (e.g., Carpenter et al., 2000; Milos et al., 2004; Eichen et al., 2016). Using such brief measures is problematic, as it makes it difficult to capture accurate levels of suicidal ideation, potentially leading to an underestimation of suicidal ideation (Glenn \& Klonsky, 2012). Suicidal ideation is a complex, dynamic experience and assessments of suicidal ideation should capture its complexity, which I aimed to do in the current study.

\section{The Present Study}

In sum, there is a paucity of research on suicidal ideation in EDs. The studies that do exist have significant shortcomings, making it difficult to test theoretical explanations of the relationships. The proposed study investigated the ED-suicidal ideation link by examining longitudinal relations between ED symptoms and suicidal ideation. First, I tested if ED symptoms and suicidal ideation related to each other at each time point. Given the robust association found in cross-sectional work (Chesney, Goodwin, \& Fazel, 2014), I hypothesized that they would be related at each time point. Additionally, I tested whether ED symptoms and suicidal ideation predicted one another over time. I hypothesized that after controlling for 
baseline suicidal ideation, ED symptoms would longitudinally predict increased suicidal ideation. Moreover, I hypothesized that after controlling for baseline ED symptoms, suicidal ideation would longitudinally predict increased ED symptoms. Overall, I predicted that a reciprocal relationship would exist between suicidal ideation and ED symptoms as there is support that the relationship between these conditions can flow in both directions (Forrest et al., 2016; Smith, Velkoff, Riberiro, \& Franklin, 2018; Yao et al. 2016). This research was conducted using an ED patient sample assigned with DSM-5 diagnoses, used short (i.e., one week) followup periods, and relied on a more rigorous four-item measurement of suicidal ideation.

\section{Method}

The proposed study is a secondary analysis of data collected for a longitudinal study detailed in Smith et al. (2018). The hypotheses presented here are unique to this proposal. All study procedures were conducted following approval from relevant Institutional Review Boards.

\section{Participants}

Adults with EDs $(N=92)$ participated in this study. Participants were recruited from treatment facilities throughout the U.S. DSM-5 (APA, 2013) diagnostic criteria were utilized when assigning diagnoses, see Measures section for more details. Approximately one-third of the sample $(n=32)$ met criteria for AN/subthreshold AN, $40 \%$ of the sample $(n=37)$ met for $\mathrm{BN} /$ subthreshold $\mathrm{BN}$, and the rest of the sample was comprised of individuals with $\mathrm{BED} /$ subthreshold $\mathrm{BED}(n=12)$, purging disorder $(n=3)$, night eating syndrome $(n=7)$, and unspecified feeding/eating disorder $(n=1)$. The sample ranged in age from 18-62 $(M=32.35$; $S D=11.35)$, was primarily White $(93.5 \%)$, non-Hispanic $(98.9 \%)$, and mostly identified as female $(96 \%)$.

\section{Procedure}

Patients were informed of the study through a flyer while attending treatment at an ED treatment facility. Patients who indicated interest were directed to a pre-screening survey that assessed disordered eating symptoms. Only those with active symptoms were invited by e-mail to participate. A total of 110 individuals with EDs were eligible following the prescreening questionnaire and read a consent form detailing the study. Only 92 individuals provided consent and completed some or all of the baseline questionnaire and weekly follow-up assessments. Data were collected online using secure survey software at baseline and on a weekly basis for four weeks following baseline. Participants were compensated $\$ 10$ for baseline, $\$ 5$ for each weekly assessment, and a $\$ 5$ bonus for completing all four weekly assessments.

\section{Measures}

ED diagnosis. The Eating Disorder Diagnostic Scale-DSM-5 Version (EDDS-5; Stice, n.d., http://ww.orr.org/sticemeasures) is a 23-item self-report measure assessing DSM-5 ED diagnostic criteria within the past three months. The original EDDS has high internal consistency and convergent validity with the Eating Disorder Examination (EDE; Cooper \& Fairburn, 1987) among people with EDs. Additionally, the EDDS is both sensitive to and predictive of changes in ED symptoms during ED treatment (Stice, Fisher, \& Martinez, 2004). Items on the scale vary in form. Question types include: Likert scale (0-6) questions (e.g., over the past three months, have you felt fat?), binary "yes/no" response questions (e.g., did you eat alone because you were embarrassed by how much you were eating?), and frequency estimation questions (e.g., how many times per month on average over the past 3 months have you fasted?).

Symptoms reported by participants on the EDDS-5 were corroborated against other ED symptom reports and body mass index (BMI) by three of the authors in the Smith et al. (2018) 
paper. Any disagreements were discussed until consensus was reached regarding which ED diagnosis participants should be assigned.

ED symptoms. ED symptoms were additionally assessed using the Eating Disorder Examination-Questionnaire, 4th edition (EDE-Q; Fairburn \& Beglin, 1994) which is based on the EDE, a structured interview (Cooper \& Fairburn, 1987). Research suggests that the EDE-Q may be used in place of investigator-based interviews (Fairburn \& Beglin, 1994). The EDE-Q consists of 36 items and four subscales that assess behavior and attitudes with regard to: 1) eating concerns, 2) shape concerns, 3) weight concerns, and 4) restraint across the past 28 days. At baseline, the EDE-Q was used in its original form and assessed ED symptoms experienced in the past 28 days. When the EDE-Q was administered over the weekly follow-ups, questions were altered to assess ED symptoms experienced in the past seven days. The EDE-Q also asks frequency questions about the following behaviors; overeating, binge eating (overeating with the presence of guilt), purging, laxative usage, diuretics usage, and compulsive exercising. Although examining these behaviors within the proposed model has to the potential of identifying specific behaviors that may influence or be influenced by the occurrence of suicidal ideation, I refrained from running these analyses as endorsement in these behaviors was relatively low. Specifically, the means across study time points for the number of instances participants engaged in these behaviors ranged from .64-.79 for purging, .01-.35 for laxative usage, 1.01-1.37 for binging, and .94-1.14 for compulsively exercising. Reliability in the present sample was acceptable to good across time points for the global score $(\alpha=.82-.88)$, eating concerns subscale $(\alpha=.71-.81)$, shape concerns subscale $(\alpha=.83-.91$.), weight concerns subscale $(\alpha=.72-.78)$, and restraint subscale $(\alpha=.87-.90)$.

Suicidal ideation. Suicidal ideation was assessed using the Depressive Symptom Inventory Suicidality Subscale (Joiner et al., 2002; Metalsky \& Joiner, 1997). This measure includes four questions assessing whether a participant experienced suicidal thoughts in the past week. The scale includes items that assess the frequency of ideation, whether participants had planned a suicide attempt, the ability to maintain control over suicidal thoughts, and the intensity of impulses to attempt suicide. Each item includes descriptions of four levels of severity for the facets of suicide ideation and desire, which ranged in score from 0 to 3 (with greater scores indicating more severity). The four items are summed for a total score (range from 0-12). Construct validity is high as it has been shown to significantly correlate with the Beck Depression Inventory (Beck, Steer, Ball, \& Ranieri, 1996; von Glischinski et al., 2016) and general emotional distress (Joiner et al., 2002). Reliability in the present sample was good across time points $(\alpha=.87-89)$.

\section{Analytic Strategy}

Data were analyzed using SPSS version 24.0 and Mplus version 7.3 (Muthén \& Muthén, 1998-2012).

Missing data. Little's missing completely at random (MCAR) test was used to examine the pattern of missing data. The test was non-significant $\left(\chi^{2}=84.19\right.$, $\left.\mathrm{df}=72, p=.15\right)$, indicating that these data were consistent with a pattern of data missing completely at random. Pairwise deletion was used to present and examine the bivariate correlations between study variables, which are presented in Table 1. The structural models were tested in Mplus version 7.3 using full-information maximum likelihood (FIML) to handle missing data.

Data screening. To identify whether any variables were non-normally distributed, skew and kurtosis indexes were computed; all ED symptom variables and most suicidal ideation variables had acceptable levels of skew and kurtosis $<|2|$. Because the suicidal ideation variables 
were zero-inflated, I considered a binary transformation (i.e., if the individual endorsed any form of suicidal ideation, they were given a score of 1 while individuals denying suicidal ideation received a score of 0 ). While this adjustment increased the overall fit of the model, it was not a significant improvement (see Table 2 for information on model fit). Since one of the aims of this study was to use a more rigorous measure of suicidal ideation (i.e., employ a continuous measure), the analyses preceded without using the binary method.

Path analyses. A sample size of 92 is below typical "rule of thumb" recommendations for structural equation modeling, which suggest sample sizes of approximately 200 (Hoogland \& Boomsma, 1998; Boomsma \& Hoogland, 2001). However, this recommendation is believed to be overly conservative and not generalizable to all studies (Iacobucci, 2010). Further, some work suggests that smaller samples with well-developed models and more liberal power estimates (e.g., .80) can still allow for SEMs to be responsibly run (Kim, 2005); thus, the study moved forward with testing the proposed model. Four primary paths were considered: (1) the autoregressive path of ED symptoms at one time point (T) predicting ED symptoms at the next time point $(\mathrm{T}+1),(2)$ the autoregressive path of suicidal ideation at $\mathrm{T}$ predicting suicidal ideation at $\mathrm{T}+1,(3)$ the cross-lagged path of ED symptoms at $\mathrm{T}$ predicting suicidal ideation at $\mathrm{T}$ +1 , and (4) the cross-lagged path of suicidal ideation at $\mathrm{T}$ predicting ED symptoms at $\mathrm{T}+1$ (see Figure 1). Because the assumption was that the relation between ED symptoms across waves would be the same, these paths were constrained to be equal. The same was done with the suicidal ideation variables. The relative strengths of the longitudinal relationships were determined through comparison of unstandardized betas.

\section{Results}

\section{Hypothesis 1: ED Symptoms and Suicidal Ideation will Relate to Each Other at Each Time Point}

The first aim was to replicate previous findings and determine if ED symptoms and suicidal ideation related to each other at each time point. Intercorrelations among variables in Table 1 help to answer this question. Correlations ranged in strength from .25-.36 and were significant at all time points. Notably, this relationship remained significant even though there was a decrease in the endorsement of both symptoms during the course of the study.

\section{Hypotheses 2 and 3: Suicidal Ideation will Predict ED Symptoms at the next Time Point and Vice Versa}

The second aim was to determine if ED symptoms or suicidal ideation predicted the other at the following time point. In order to examine this aim, an autoregressive cross-lag model was used to analyze how suicidal ideation and ED symptomatology affect each other over the course of the five-week study period (see Figure 1). Model fit was assessed using the comparative fit index (CFI; Bentler, 1990), the Tucker-Lewis index (TLI; Tucker \& Lewis, 1973), the root mean square error of approximation (RMSEA; Browne \& Cudeck, 1993), and the standardized root mean square residual (SRMR; Hu \& Bentler, 1999). Since CFI and TLI values were above .90 $(\mathrm{CFI}=.95, \mathrm{TLI}=.93)$ but RMSEA and SMR were not below $0.05(\mathrm{RMSEA}=.10, \mathrm{SMR}=.09)$, model fit ranged from mediocre to acceptable. The majority of the pathways in our model were non-significant, indicating that (1) other variables may better explain the relation between these variables, (2) I was underpowered to detect effects due to small sample size, or (3) the variables were too strongly related to each other across time points (e.g., Week 1 ED symptoms and Week 2 ED symptoms) to leave enough predictive power for another variable. However, results did reveal one weak, but statistically significant cross-lagged path: suicidal ideation at Week 3 predicted ED symptoms at Week 4 while controlling for Week 3 ED symptoms $(B=0.06$, S.E. $=$ 
$.07, p<.05)$. Additional analyses were conducted by substituting the EDE-Q Global score with each EDE-Q subscale into the cross-lagged model.

Eating concerns. Model fit ranged from poor to acceptable (model fit indices are reported in Table 2). There were no significant cross-lagged pathways, although one pathway suicidal ideation at Week 3 predicting eating concerns at Week $4(B=0.07$, S.E. $=.04, p=.059)$ - trended towards significance.

Shape concerns. Model fit ranged from mediocre to good (model fit indices can be found in Table 2). The only significant cross-lagged pathway was suicidal ideation at Week 3 predicting shape concerns at Week $4(B=0.08$, S.E. $=.03, p<.05)$. However, two other crosslagged pathways trended towards significance. These paths were suicidal ideation at Week 1 predicting shape concerns at Week $2(B=0.05$, S.E. $=.03, p=.053)$, and shape concerns at Week 1 predicting suicidal ideation at Week $2(B=0.17$, S.E. $=.09, p=.058)$. Although not statistically significant, this was one instance where some evidence for a reciprocal relationship between suicidal ideation and an ED symptom was found.

Weight concerns. Model fit ranged from poor to acceptable (model fit indices are reported in Table 2). The significant cross-lagged pathways were weight concerns at Week 1 predicting suicidal ideation at Week $2(B=0.17$, S.E. $=.04, p<.05)$, as well as suicidal ideation at Week 3 predicting weight concerns at Week $4(B=0.07$, S.E. $=.04, p<.05)$.

Restraint. Model fit was poor on all model fit indices, see Table 2. No significant crosslagged pathways were found with suicidal ideation predicting restraint and vice versa.

\section{Discussion}

Longitudinal research examining the association between EDs and suicidal ideation is essentially non-existent. This study tested whether ED symptoms and suicidal ideation were related to and could predict one another at weekly time points over the course of a month. Supporting my first hypothesis, I found that these symptoms remained correlated with each other at each time point over the duration of the study. This finding, along with previous research that also found a robust association between suicidal ideation and EDs (Chesney, Goodwin, \& Fazel, 2014), underscores the importance of assessing suicidal ideation in ED populations.

Unfortunately, the assessment and treatment of suicidal ideation is not routinely done by clinicians working with individuals with EDs; research using 1,432 ED patients found that providers screened for STBs in less than $50 \%$ of all cases (Peebles, Wilson, \& Lock, 2010). In order to continue to understand the association between ED symptoms and suicidal ideation, an important next step is determining the directionally of the suicidal ideation-ED relationship.

Although there exists an assumption that EDs serve as a risk factor for - and therefore, may precede - suicidal thoughts and behaviors, the results from this study illustrated another picture. The one significant cross-lagged pathway in my study actually indicated that suicidal ideation can predict general ED symptoms at a following time point. In other words, if someone is experiencing thoughts about suicide, they might be more likely to engage in ED symptoms in the near future. However, the majority of cross-lagged paths were not significant. While these results were only partially supportive of my hypothesis that a reciprocal relationship exists, they have clinically significant implications. This finding suggests that ED symptoms may be used to cope with suicidal thoughts. If this is the case, it would be pertinent for clinicians working with patients experiencing suicidality to identify adaptive coping strategies that can help patients manage distressing thoughts without putting them at risk for experiencing other forms of psychopathology (i.e., ED symptoms). 
Relapse rates for EDs are high (Berkman et al., 2006; Carter, Blackmore, SuntandarPinnock, \& Woodside, 2004). The finding that suicidal ideation might lead to future engagement of ED symptoms may offer a small, but novel, explanation for why this is the case. First-line psychotherapeutic treatments for EDs often include cognitive behavioral interventions, which target issues related to food, shape, and weight. Although such interventions may be effective in reducing ED symptoms, they may not be able to target all of the psychopathology that leads an individual to engage in those symptoms. Traditionally, ED treatment efforts, especially for anorexia nervosa, have neglected the role of emotion regulation in the development and maintained of EDs (Haynos \& Fruzzetti, 2011). While some treatment approaches acknowledge that those with EDs often have difficulty identifying and expressing emotions accurately (Garner, Vitousek, Pike, \& Garfinkel, 1997), most work has not focused on helping individuals tolerate and effectively regulate emotional arousal (e.g., Fairburn, Cooper, \& Shafarn, 2003). Thus, if an individual in recovery from their ED begins to experience increases in suicidal thoughts, they may not have developed adaptive emotion regulation strategies and could revert back to disordered eating habits. Therefore, my results underscore the importance of evaluating and correcting strategies used to manage suicidal thoughts as it may decrease ED relapse in the future.

In addition to testing a model with the global EDE-Q score, I tested the subscales of the EDE-Q to better understand whether specific ED cognitions and symptomatology might be driving the relationship. When weight concerns, shape concerns, and eating concerns were individually entered into the model, I found a similar trend as with the EDE-Q global score where suicidal ideation at Week 3 predicted ED symptoms at Week 4. This result contradicts some previous research. For instance, Kim and Kim (2009) found that body dissatisfaction prospectively predicted suicidal ideation in males and females. However, a limitation of this study is there was a two-year gap between time points so it was not able to capture earlier fluctuations in the relationship between these variables. It was somewhat surprising that I did not find any significant cross-lagged pathways for restraint predicting ideation as previous work has found that restraint is related to elevated reports of suicidal thoughts within an ED sample (Forrest et al., 2016). However, another study also failed to find an association between dietary restraint and suicidal ideation in a sample of undergraduate students (Zuromski \& Witte, 2015). It should be explored if specific components of suicidal desire, such as low belongingness or perceived burdensomeness, better explain the longitudinal association between restraint and suicidal ideation.

Given the limited number of statistically significant pathways with the models, it is possible that shared risk factors, or "third variables" may better explain the relation between ED symptoms and suicidal ideation. Given that the average age of onset of AN, BN, and suicidal ideation are roughly similar (around 16-18 for AN and BN behaviors [Mustelin et al., 2016; Stice, Killen, Hayward, \& Taylor, 1998] and 13-15 for suicide suicidal ideation [Kerr, Owen, Pears, \& Capaldi, 2008; Rueter \& Kwon, 2005]), this is certainly a possibility. Of note, Yao and colleagues (2016) found that the co-occurrence of other mental disorders (e.g. major depressive disorder, anxiety disorders, substance abuse disorders) did not fully explain the relationship between EDs and STBs, suggesting that these shared mechanisms alone cannot account for the co-occurrence of these conditions. Other possible third variables to consider including in future longitudinal research studies are interoceptive deficits (i.e., the inability to recognize and correctly interpret one's bodily sensations) and self-criticism as these variables have found to be related to both suicidal ideation (e.g., Forrest, Smith, White, \& Joiner, 2015; O’Conner \& Noyce, 
2008) and EDs (e.g., Fennig et al., 2008; Merwin, Zucker, Lacy, \& Elliott, 2010). Overall, it is pertinent that we continue to investigate why those engaging in disordered eating behaviors are at increased risk for experiencing suicidal ideation in order to identify ways to disrupt this association.

\section{Strengths, Limitations, and Future Directions}

The present findings should be interpreted in conjunction with the study's strengths and limitations, which each inform directions for future research. With respect to strengths, this study used longitudinal methodology with shorter follow-up points compared to previous research (Bodell, Cheng, \& Wildes, 2018). Additionally, I used DSM-5 diagnostic criteria and a more sensitive measure of suicidal ideation. It is important to study suicide risk among people with EDs from a transdiagnostic perspective. I achieved this in the current study by including participants that represented most of the DSM-5 ED diagnoses.

With respect to limitations, the sample size may have impacted my ability to detect crosslagged relationships. Future research would benefit from using larger sample sizes when exploring how ED symptomatology and suicidality variables affect one another over time. An additional limitation was that I did not have information on what phase of treatment these individuals were in or if they were still enrolled in treatment by study end. Since it is common to see large improvements during the first half of treatment (MacDonald, Trottier, McFarlane, \& Olmsted, 2015; Turner, Bryant-Waugh, \& Marshall, 2015), that could have impacted the associations I found. Future research with treatment seeking samples should gather information about the stage of treatment patients are in.

While this study was an important first step in understanding the association between EDs and suicidal ideation, future studies should test these relationships within a community sample. There are multiple benefits to this. First, research has found that recruited clinical samples are not fully representative of those who meet full criteria for an ED. Specifically, recruited clinical samples tend to be younger, predominately white, and higher educated compared to recruited community samples (Wilfley et al., 2001). Given that there are racial/ethnic differences in the presentation of ED symptoms, a more diverse community sample might endorse greater shape, weight, and eating concerns (Franko et al., 2012), as well as purging behaviors (Franko, Becker, Thomas, \& Herzog, 2007). Second, I might have seen patterns that are unique to patients being in treatment (i.e., a decrease in symptom endorsement over the course of the study), so it would be interesting to see if different fluctuations exist within a community sample.

Future work should also consider using even shorter follow-up points. Ecological momentary assessment (EMA), a technique that captures participants' subjective experiences at random time points over a day or week, is likely to be more sensitive to contextual variables that impact urges such as location, time of day, and mood (e.g., negative affect). While EMA has been largely neglected in the field of suicidology (Davidson, Anestis, \& Gutierrez, 2017), using this method to examine short-term fluctuations in the relationship between ED symptoms and suicidal ideation can help to identify more immediate factors that may be influencing this association.

\section{Conclusion}

The robust association between EDs and suicide have led many to assume that EDs are risk factors for suicide outcomes; yet, few longitudinal studies have tested this prediction. While my hypothesis that a reciprocal relationship exists was only partially supported, it contradicted the general assumption that is made by the research field. Specifically, the only consistent 
longitudinal influence these variables had on each other in my study was an instance where suicidal ideation predicted future engagement in ED symptoms. More specifically, suicidal ideation predicted endorsement of cognitions related to concerns about weight, shape, and eating (but not restraint) at the following week. These findings highlight that thoughts about suicide can contribute to ED symptoms. Given that suicidal ideation may influence future engagement in ED symptoms, targeting suicidal thoughts in therapy may help clinicians reduce eating pathology in their ED patients. 
References

Almeida, L., Savoy, S., \& Boxer, P. (2011). The role of weight stigmatization in cumulative risk for binge eating. Journal of Clinical Psychology, 67, 278-292.

American Psychiatric Association. (2013). Diagnostic and statistical manual of mental disorders, 5th edition: DSM-5. Arlington, VA: American Psychiatric Publising Incorporated.

Arcelus, J., Mitchell, A. J., Wales, J., \& Nielsen, S. (2011). Mortality rates in patients with anorexia nervosa and other eating disorders: a meta-analysis of 36 studies. Archives of General Psychiatry, 68(7), 724-731.

Ashmore, J. A., Friedman, K. E., Reichmann, S. K., \& Musante, G. J. (2008). Weight-based stigmatization, psychological distress, and binge eating behavior among obese treatmentseeking adults. Eating Behaviors, 9(2), 203-209.

Bearman, S. K., Presnell, K., Martinez, E., \& Stice, E. (2006). The skinny on body dissatisfaction: A longitudinal study of adolescent girls and boys. Journal of Youth and Adolescence, 35(2), 217-229.

Beck, A. T., Steer, R. A., Ball, R., \& Ranieri, W. (1996). Comparison of Beck Depression Inventories-IA and -II in psychiatric outpatients. Journal of Personality Assessment, 67(3), 588-597. http://doi.org/10.1207/s15327752jpa6703_13

Bentler, P. M. (1990). Comparative fit indexes in structural models. Psychological Bulletin, 107(2), 238.

Berkman, N. D., Bulik, C. M., Brownley, K. A., Lohr, K. N., Sedway, J. A., Rooks, A., \& Gartlehner, G. (2006). Management of eating disorders. Agency for Healthcare Research and Quality, 135, 1-166.

Bodell, L. P., Cheng, Y., \& Wildes, J. E. (2018). Psychological impairment as a predictor of suicide ideation in individuals with anorexia nervosa. Suicide and Life-Threatening Behavior.

Bohn, K., Doll, H. A., Cooper, Z., O'Connor, M., Palmer, R. L., \& Fairburn, C. G. (2008). The measurement of impairment due to eating disorder psychopathology. Behaviour Research and Therapy, 46(10), 1105-1110.

Boomsma, A. \& Hoogland, J. J. (2001). The robustness of LISREL modeling revisited. Structural equation models: Present and future. A Festschrift in honor of Karl Jöreskog, 2(3), 139-168.

Browne, M. W. \& Cudeck, R. (1993). Alternative ways of assessing model fit. Sage focus editions, 154, 136-136.

Carpenter, K. M., Hasin, D. S., Allison, D. B., \& Faith, M. S. (2000). Relationships between obesity and DSM-IV major depressive disorder, suicide ideation, and suicide attempts: results from a general population study. American journal of public health, 90(2), 251.

Chesney, E., Goodwin, G. M., \& Fazel, S. (2014). Risks of all-cause and suicide mortality in mental disorders: A meta-review. World Psychiatry, 13, 153-160.

Cooper, Z., \& Fairburn, C. (1987). The eating disorder examination: A semi-structured interview for the assessment of the specific psychopathology of eating disorders. International Journal of Eating Disorders, 6(1), 1-8.

Cooper, M. J., Wells, A., \& Todd, G. (2004). A cognitive model of bulimia nervosa. British Journal of Clinical Psychology, 43(1), 1-16. 
Crow, S. J., Swanson, S. A., Le Grange, D., Feig, E. H., \& Merikangas, K. R. (2014). Suicidal behavior in adolescents and adults with bulimia nervosa. Comprehensive Psychiatry, 55(7), 1534-1539.

Davidson, C. L., Anestis, M. D., \& Gutierrez, P. M. (2017). Ecological momentary assessment is a neglected methodology in suicidology. Archives of suicide research, 21(1), 1-11.

Dodd, D. R., Smith, A. R., \& Bodell, L. P. (2014). Restraint feeds stress: the relationship between eating disorder symptoms, stress generation, and the interpersonal theory of suicide. Eating Behaviors, 15, 567-573.

Eichen, D. M., Kass, A. E., Fitzsimmons-Craft, E. E., Gibbs, E., Trockel, M., Taylor, C. B., \& Wilfley, D. E. (2016). Non-suicidal self-injury and suicidal ideation in relation to eating and general psychopathology among college-age women. Psychiatry Research, 235, 7782.

Fairburn, C., Cooper, Z., \& Shafran, R. (2003). Cognitive behaviour therapy for eating disorders: A "transdiagnostic'" theory and treatment. Behavior Research and Therapy, 41, 509528.

Fairburn, C. G. \& Beglin, S. J. (1994). Assessment of eating disorders: Interview or self-report questionnaire?. International Journal of Eating Disorders, 16(4), 363-370.

Favaro, A., \& Santonastaso, P. (1997). Suicidality in eating disorders: Clinical and psychological correlates. Acta Psychiatrica Scandinavica, 95(6), 508-514.

Fennig, S., Hadas, A., Itzhaky, L., Roe, D., Apter, A., \& Shahar, G. (2008). Self-criticism is a key predictor of eating disorder dimensions among inpatient adolescent females. International Journal of Eating Disorders, 41(8), 762-765.

Fergusson, D. M., Horwood, L. J., Ridder, E. M., \& Beautrais, A. L. (2005). Suicidal behaviour in adolescence and subsequent mental health outcomes in young adulthood. Psychological Medicine, 35(7), 983-993.

Franko, D. L., Becker, A. E., Thomas, J. J., \& Herzog, D. B. (2007). Cross-ethnic differences in eating disorder symptoms and related distress. International Journal of Eating Disorders, 40(2), 156-164.

Franko, D. L., Thompson-Brenner, H., Thompson, D. R., Boisseau, C. L., Davis, A., Forbush, K. T., ... Wilson, G. T. (2012). Racial/ethnic differences in adults in randomized clinical trials of binge eating disorder. Journal of Consulting and Clinical Psychology, 80(2), 186-195.

Frisch, M. J., Herzog, D. B., \& Franko, D. L. (2006). Residential treatment for eating disorders. International Journal of Eating Disorders, 39, 434-442.

Forrest, L. N., Bodell, L. P., Witte, T. K., Goodwin, N., Bartlett, M. L., Siegfried, N., ... \& Smith, A. R. (2016). Associations between eating disorder symptoms and suicidal ideation through thwarted belongingness and perceived burdensomeness among eating disorder patients. Journal of Affective Disorders, 195, 127-135.

Forrest, L. N., Smith, A. R., \& Swanson, S. A. (2017). Characteristics of seeking treatment among US adolescents with eating disorders. International Journal of Eating Disorders, $50,826-833$.

Forrest, L. N., Smith, A. R., White, R. D., \& Joiner, T. E. (2015). (Dis) connected: An examination of interoception in individuals with suicidality. Journal of Abnormal Psychology, 124(3), 754. 
Forrest, L. N., Zuromski, K. L., Dodd, D. R., \& Smith, A. R. (2017). Suicidality in adults and adolescents with binge eating disorder: Results from the National Comorbidity Survey Replication and Adolescent Supplement. International Journal of Eating Disorders.

Franklin, J. C., Ribeiro, J. D., Fox, K. R., Bentley, K. H., Kleiman, E. M., Huang, X., ... \& Nock, M. K. (2017). Risk factors for suicidal thoughts and behaviors: A meta-analysis of 50 years of research. Psychological Bulletin, 143(2), 187.

Garner, D. M., Vitousek, K. M., Pike, K. M., \& Garfinkel, P. E. (1997). Cognitive-behavioral therapy for anorexia nervosa. In D. M. Garner \& P. E. Garfinkel (Eds.), Handbook of treatment for eating disorders (2nd ed., pp. 94-144). New York: Guilford Press.

Glenn, C.R. \& Klonsky, D.E. (2012, November). Factors to consider in the assessment of suicidal thoughts and behaviors in adolescents. Symposium conducted at the meeting of Association for Behavioral and Cognitive Therapy, National Harbor, Maryland.

Harney, M. B., Fitzsimmons-Crafr, E. E., Maldonado, C. R., \& Bardone-Cone, A. M. (2014). Negative affect experiences in relation to states of eating disorder recovery. Eating Behaviors, 15(1), 24-30.

Harrison, A., Mountford, V. A., \& Tchanturia, K. (2014). Social anhedonia and work and social functioning in the acute and recovered phases of eating disorders. Psychiatry Research, 218, 187-194.

Hawton, K. \& van Heeringen, K. (2009) Suicide. The Lancet, 373, 18-24. DOI: 10.1016/S01406736(09)60372-X

Haynos, A. F., \& Fruzzetti, A. E. (2011). Anorexia nervosa as a disorder of emotion dysregulation: Evidence and treatment implications. Clinical Psychology: Science and Practice, 18, 183-202.

Heatherton, T. F. \& Baumeister, R. F. (1991). Binge eating as escape from self-awareness. Psychological Bulletin, 110(1), 86.

Hoogland, J. J. \& Boomsma, A. (1998). Robustness studies in covariance structure modeling: An overview and a meta-analysis. Sociological Methods \& Research, 26(3), 329-367.

Hu, L. T. \& Bentler, P. M. (1999). Cutoff criteria for fit indexes in covariance structure analysis: Conventional criteria versus new alternatives. Structural Equation Modeling: A Multidisciplinary Journal, 6(1), 1-55.

Iacobucci, D. (2010). Structural equations modeling: Fit indices, sample size, and advanced topics. Journal of Consumer Psychology, 20(1), 90-98.

Joiner, T. E. (2005). Why people die by suicide. Cambridge, MA: Harvard University Press.

Joiner Jr, T. E., Pfaff, J. J., \& Acres, J. G. (2002). A brief screening tool for suicidal symptoms in adolescents and young adults in general health settings: Reliability and validity data from the Australian National General Practice Youth Suicide Prevention Project. Behaviour Research and Therapy, 40(4), 471-481.

Kerr, D. C. R., Owen, L. D., Pears, K. C., \& Capaldi, D. M. (2008). Prevalence of suicidal ideation among boys and men assessed annually from ages 9 to 29 years. Suicide and Life-Threatening Behavior, 38(4), 390-402.

Keys, A., Borzek, J., Henschel, A., Mickelsen, O., \& Taylor, H. L. (1950). The biology of human starvation (volume 2). Minneapolis, MN: University of Minnesota Press.

Kim, K. H. (2005). The relation among fit indexes, power, and sample size in structural equation modeling. Structural Equation Modeling, 12(3), 368-390. 
Kim, D. S., \& Kim, H. S. (2009). Body-image dissatisfaction as a predictor of suicidal ideation among Korean boys and girls in different stages of adolescence: A two-year longitudinal study. Journal of Adolescent Health, 45(1), 47-54.

Kline, R. B. (2011). Principles and practice of structural equation modeling (3rd ed.). New York, NY: Guilford Press.

Leehr, E. J., Krohmer, K., Schag, K., Dresler, T., Zipfel, S., \& Giel, K. E. (2015). Emotion regulation model in binge eating disorder and obesity - A systematic review. Neuroscience \& Biobehavioral Reviews, 49, 125-134. https://doi.org/10.1016/j.neubiorev.2014.12.008

Linardon, J., Brennan, L., \& De la Piedad Garcia, X. (2016). Rapid response to eating disorder treatment: A systematic review and meta-analysis. International Journal of Eating Disorders, 49(10), 905-919.

Links, P. S., Eynan, R., Heisel, M. J., Barr, A., Korzekwa, M., McMain, S., \& Ball, J. S. (2007). Affective instability and suicidal ideation and behavior in patients with borderline personality disorder. Journal of Personality Disorders, 21, 72-86.

MacDonald, D. E., Trottier, K., McFarlane, T., \& Olmsted, M. P. (2015). Empirically defining rapid response to intensive treatment to maximize prognostic utility for bulimia nervosa and purging disorder. Behaviour Research and Therapy, 68, 48-53.

Merwin, R. M., Zucker, N. L., Lacy, J. L., \& Elliott, C. A. (2010). Interoceptive awareness in eating disorders: Distinguishing lack of clarity from non-acceptance of internal experience. Cognition and Emotion, 24(5), 892-902.

Metalsky, G. I. \& Joiner, T. E. (1997). The hopelessness depression symptom questionnaire. Cognitive Therapy and Research, 21(3), 359-384.

Milos, G., Spindler, A., Hepp, U., \& Schnyder, U. (2004). Suicide attempts and suicidal ideation: Links with psychiatric comorbidity in eating disorder subjects. General Hospital Psychiatry, 26(2), 129-135.

Mustelin, L., Silén, Y., Raevuori, A., Hoek, H. W., Kaprio, J., \& Keski-Rahkonen, A. (2016). The DSM-5 diagnostic criteria for anorexia nervosa may change its population prevalence and prognostic value. Journal of Psychiatric Research, 77, 85-91.

Muthén, L. K. \& Muthén, B. O. (1998-2012). Mplus User's Guide: Statistical Analysis with Latent Variables (7th ed.). Los Angeles, CA: Muthén \& Muthén.

Nock, M. K., Borges, G., Bromet, E. J., Cha, C. B., Kessler, R. C., \& Lee, S. (2008). Suicide and suicidal behavior. Epidemiologic Reviews, 30, 133-154.

Nock, M. K., Prinstein, M. J., \& Sterba, S. K. (2009). Revealing the form and function of selfinjurious thoughts and behaviors: a real-time ecological assessment study among adolescents and young adults. Journal of Abnormal Psychology, 118, 816-827.

O’Connor, R. C., \& Noyce, R. (2008). Personality and cognitive processes: Self-criticism and different types of rumination as predictors of suicidal ideation. Behaviour Research and Therapy, 46(3), 392-401.

Paxton, S. J. \& Diggens, J. (1997). Avoidance coping, binge eating, and depression: An examination of the escape theory of binge eating. International Journal of Eating Disorders, 22(1), 83-87.

Pisetsky, E. M., Crow, S. J., \& Peterson, C. B. (2017). An empirical test of the interpersonal theory of suicide in a heterogeneous eating disorder sample. International Journal of Eating Disorders, 50(2), 162-165. 
Rieger, E., van Buren, D. J., Bishop, M., Tanofsky-Kraff, M., Welch, R., \& Wilfley, D. E. (2010). An eating disorder-specific model of interpersonal psychotherapy (IPT-ED): Causal pathways and treatment implications. Clinical Psychology Review, 30, 400-410.

Rodziński, P., Rutkowski, K., \& Ostachowska, A. (2017). Progression of suicidal ideation to suicidal behavior from a perspective of selected suicidological models. Psychiatria Polska, 51(3), 515-530.

Rueter, M. A. \& Kwon, H. (2005). Developmental trends in adolescent suicidal ideation. Journal of Research on Adolescence, 15, 205-222.

Samnaliev, M., Noh, H. L., Sonneville, K. R., \& Austin, S. B. (2015). The economic burden of eating disorders and related mental health comorbidities: an exploratory analysis using the U.S. Medical Expenditures Panel Survey. Preventative Medicine Reports, 2, 32-34.

Schmidt, U. \& Treasure, J. (2006). Anorexia nervosa: Valued and visible. A cognitiveinterpersonal maintenance model and its implications for research and practice. British Journal of Clinical Psychology, 45(3), 343-366.

Selby, E. A., Smith, A. R., Bulik, C. M., Olmsted, M. P., Thornton, L., McFarlane, T. L.,...\& Joiner, T. E. Jr. (2010). Habitual starvation and provocative behaviors: Two potential routes to extreme suicidal behavior in anorexia nervosa. Behavior Research and Therapy, 48, 364-645.

Sepulveda, A. R., Anastasiadou, D., Pellegrin, Y., Andrés, P., Graell, M., Carrobles, J. A., \& Morandé, G. (2014). Impact of caregiving experience on mental health among caregivers: a comparison of eating disorder patients with purging and non-purging behaviors. Eating and Weight Disorders, 19, 31-39.

Smith, A. R., Dodd, D. R., Forrest, L. N., Witte, T. K., Bodell, L., Ribeiro, J. D., ... \& Bartlett, M. (2016). Does the interpersonal-Psychological theory of suicide provide a useful framework for understanding suicide risk among eating disorder patients? A test of the validity of the IPTS. International Journal of Eating Disorders, 49(12), 1082-1086.

Smith, A. R., Velkoff, E. A., Ribeiro, J. D., \& Franklin, J. (2018). Are eating disorders and related symptoms risk factors for suicidal thoughts and behaviors? A meta-analysis. Suicide and Life-Threatening Behavior.

Smith, A. R., Yeager, A. E., \& Dodd, D. R. (2015). The joint influence of acquired capability for suicide and stoicism on over-exercise among women. Eating Behaviors, 17, 77-82.

Smith, A. R., Forrest, L. N., Velkoff, E. A., Ribeiro, J. D., \& Franklin, J. (2018). Implicit attitudes toward eating stimuli differentiate eating disorder and non-eating disorder groups and predict eating disorder behaviors. International Journal of Eating Disorders.

Smith, A. R., Zuromski, K. L., \& Dodd, D. R. (2018). Eating disorders and suicidality: What we know, what we don't know, and suggestions for future research. Current Opinion in Psychology, 22, 63-67.

Stice, E., Fisher, M., \& Martinez, E. (2004). Eating disorder diagnostic scale: Additional evidence of reliability and validity. Psychological Assessment, 16(1), 60.

Stice, E., Killen, J. D., Hayward, C., \& Taylor, C. B. (1998). Age of onset for binge eating and purging during late adolescence: A 4-year survival analysis. Journal of Abnormal Psychology, 107(4), 671.

Stice, E., Presnell, K., \& Spangler, D. (2002). Risk factors for binge eating onset in adolescent girls: a 2-year prospective investigation. Health Psychology, 21(2), 131-138.

Stice, E., \& Whitenton, K. (2002). Risk factors for body dissatisfaction in adolescent girls: a longitudinal investigation. Developmental Psychology, 28(5), 669-678. 
Troop, N. A., Allan, S., Serpell, L., \& Treasure, J. L. (2008). Shame in women with a history of eating disorders. European Eating Disorders Review, 16(6), 480-488.

Trujillo, A., Forrest, L., Claypool, H., Smith, A. (Under review). Assessing the relationship among thwarted belongingness, perceived burdensomeness, and eating disorder symptoms. Suicide and Life-Threatening Behavior.

Tucker, L. R. \& Lewis, C. (1973). A reliability coefficient for maximum likelihood factor analysis. Psychometrika, 38(1), 1-10.

Turner, H., Bryant-Waugh, R., \& Marshall, E. (2015). The impact of early symptom change and therapeutic alliance on treatment outcome in cognitive-behavioural therapy for eating disorders. Behaviour Research and Therapy, 73, 165-169.

Van Orden, K. A., Witte, T. K., Cukrowicz, K. C., Braithwaite, S. R., Selby, E. A., \& Joiner, T., E. (2010). The interpersonal theory of suicide. Psychological Review, 117(2), 575-600.

von Glischinski, M., Teismann, T., Prinz, S., Gebauer, J. E., \& Hirschfeld, G. (2016). Depressive Symptom Inventory Suicidality Subscale: Optimal cut points for clinical and non-clinical samples. Clinical Psychology \& Psychotherapy, 23(6), 543-549.

Wildes, J. E., Ringham, R. M., \& Marcus, M. D. (2010). Emotion avoidance in patients with anorexia nervosa: Initial test of a functional model. International Journal of Eating Disorders, 43(5), 398-404.

Wilfley, D. E., Pike, K. M., Dohm, F. A., Striegel-Moore, R. H., \& Fairburn, C. G. (2001). Bias in binge eating disorder: How representative are recruited clinic samples? Journal of Consulting and Clinical Psychology, 69(3), 383.

Zabala, M. J., Macdonald, P., \& Treasure, J. (2009). Appraisal of caregiving burden, expressed emotion and psychological distress in families of people with eating disorders: a systematic review. European Eating Disorders Review, 17, 338-349.

Zuromski, K. L. \& Witte, T. K. (2015). Fasting and the acquired capability for suicide: A test of the interpersonal-psychological theory of suicide in an undergraduate sample. Psychiatry Research, 226(1), 61-67. 
Table 1

Correlations and descriptive statistics for suicidal ideation and general eating disorder symptoms

\begin{tabular}{|c|c|c|c|c|c|c|c|c|c|c|}
\hline $\mathrm{N}=92$ & 1 & 2 & 3 & 4 & 5 & 6 & 7 & 8 & 9 & 10 \\
\hline 1. Baseline EDEQ & - & & & & & & & & & \\
\hline 2. Baseline SI & $.36 * *$ & - & & & & & & & & \\
\hline 3. Week 1 EDEQ & $.83 * *$ & $.23^{*}$ & - & & & & & & & \\
\hline 4. Week 1 SI & $.30 * *$ & $.79 * *$ & $.31 * *$ & _ & & & & & & \\
\hline 5. Week 2 EDEQ & $.82 * *$ & $.30 * *$ & $.91 * *$ & $.35 * *$ & - & & & & & \\
\hline 6. Week 2 SI & $.27 * *$ & $.66 * *$ & $.32 * *$ & $.76^{* *}$ & $.34 * *$ & - & & & & \\
\hline 7. Week 3 EDEQ & $.79 * *$ & $.23 *$ & $.85^{* *}$ & $.31 * *$ & $.88 * *$ & $.25^{*}$ & - & & & \\
\hline 8. Week 3 SI & $.24 *$ & $.71 * *$ & $.27 *$ & $.76^{* *}$ & $.32 * *$ & $.85^{* *}$ & $.35^{*}$ & - & & \\
\hline 9. Week 4 EDEQ & $.83 * *$ & $.27 *$ & $.87 * *$ & $.32 * *$ & $.90 * *$ & $.30 * *$ & $.92 * *$ & $.30 * *$ & - & \\
\hline 10. Week 4 SI & .19 & $.67 * *$ & $.25^{*}$ & $.72 * *$ & $.24 *$ & $.66^{* *}$ & $.27 *$ & $.70 * *$ & $.27 *$ & - \\
\hline Mean & 3.90 & 1.59 & 3.68 & 1.04 & 3.61 & 1.04 & 3.56 & .78 & 3.55 & .67 \\
\hline Standard deviation & 1.15 & 2.00 & 1.23 & 1.74 & 1.25 & 1.81 & 1.36 & 1.42 & 1.32 & 1.45 \\
\hline Min & 1.23 & 0.00 & .76 & 0.00 & .46 & 0.00 & .38 & 0.00 & .54 & 0.00 \\
\hline Max & 5.72 & 8.00 & 5.65 & 8.00 & 5.65 & 7.00 & 5.75 & 7.00 & 5.80 & 7.00 \\
\hline Skew & -.41 & 1.13 & -.39 & 2.08 & -.31 & 1.77 & -.32 & 1.94 & -.10 & 2.53 \\
\hline Kurtosis & -.74 & .32 & -.80 & 4.09 & -.73 & 1.97 & -.87 & 3.70 & -.91 & 6.25 \\
\hline Missing (\%) & 5.50 & 3.30 & 12.01 & 8.79 & 10.99 & 10.99 & 12.01 & 12.01 & 13.19 & 13.1 \\
\hline
\end{tabular}

Note. ${ }^{*} \mathrm{p}<.05 * * \mathrm{p}<.01 ; * * * \mathrm{p}<.001 . \mathrm{SI}=$ suicidal ideation. EDEQ = Eating Disorder Examination Questionnaire - global score 
Table 2

Model fit indices for each ED symptom entered into the model

\begin{tabular}{rllll}
\hline ED Symptom & CFI & TLI & RMSEA & SRMR \\
\hline EDE-Q - Global & .95 & .93 & .10 & .09 \\
EDE-Q - Global (SI binary) & .96 & .94 & .09 & .07 \\
Eating concerns & .94 & .92 & .11 & .09 \\
Shape concerns & .96 & .94 & .10 & .09 \\
Weight concerns & .95 & .93 & .11 & .08 \\
Restraint & .88 & .83 & .16 & .09 \\
\hline
\end{tabular}

Note. CFI = comparative fit index (Bentler, 1990); TLI = Tucker-Lewis index (Tucker \& Lewis, 1973); RMSEA = root mean square error of approximation (Browne \& Cudeck, 1993); SRMR = standardized root mean square residual (Hu \& Bentler, 1999); ED = eating disorder; EDE-Q = eating disorder examination - questionnaire; SI = suicidal ideation 


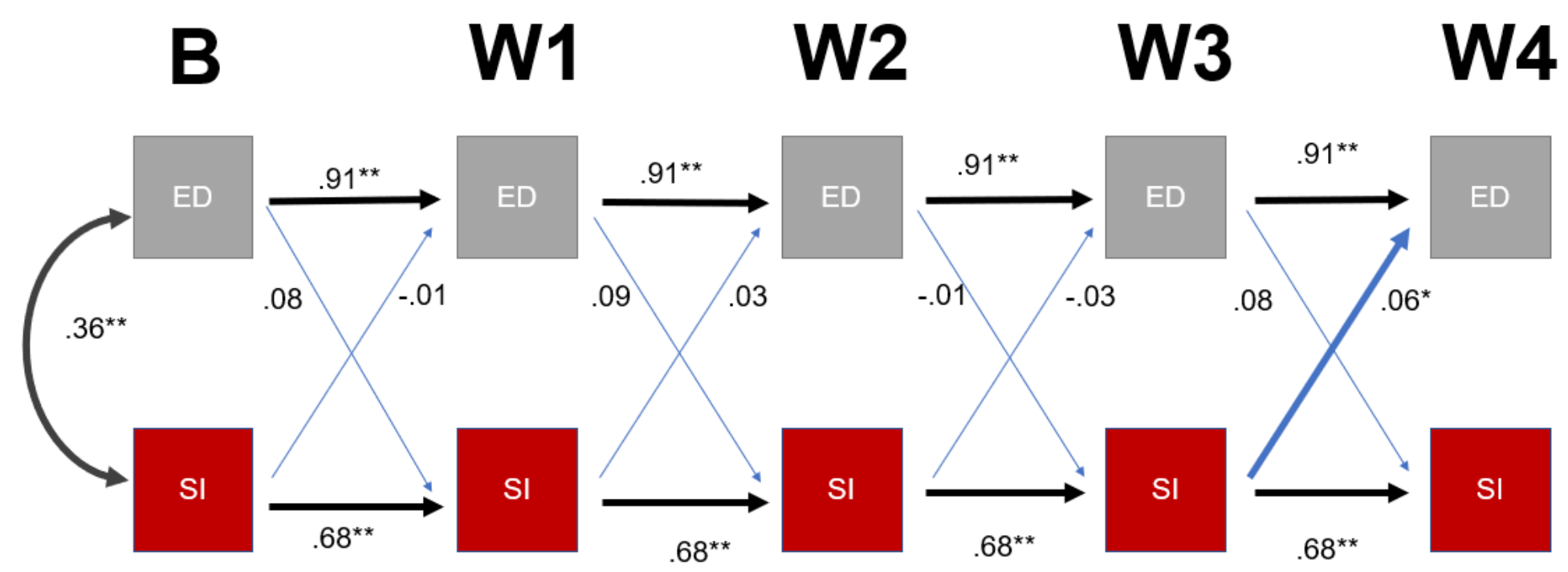

Figure 1. Unstandardized path coefficients for autoregressive cross-lag model tested. Note. $* \mathrm{p}<.05 ; * * \mathrm{p}<.01 ;$ black lines $=$ autoregressive paths; blue lines = cross-lagged paths; bolded lines = statistically significant pathways; $\mathrm{SI}=$ suicidal ideation; ED = Eating Disorder Examination Questionnaire - Global Score; B = baseline; W = week 